\title{
Partial differential equations in Banach spaces involving nilpotent linear operators
}

\author{
by Antonia Chinnì and Paolo Cubiotti (Messina)
}

Abstract. Let $E$ be a Banach space. We consider a Cauchy problem of the type

$$
\begin{cases}D_{t}^{k} u+\sum_{j=0}^{k-1} \sum_{|\alpha| \leq m} A_{j, \alpha}\left(D_{t}^{j} D_{x}^{\alpha} u\right)=f & \text { in } \mathbb{R}^{n+1}, \\ D_{t}^{j} u(0, x)=\varphi_{j}(x) & \text { in } \mathbb{R}^{n}, j=0, \ldots, k-1,\end{cases}
$$

where each $A_{j, \alpha}$ is a given continuous linear operator from $E$ into itself. We prove that if the operators $A_{j, \alpha}$ are nilpotent and pairwise commuting, then the problem is well-posed in the space of all functions $u \in C^{\infty}\left(\mathbb{R}^{n+1}, E\right)$ whose derivatives are equi-bounded on each bounded subset of $\mathbb{R}^{n+1}$.

Introduction. Let $k, m, n \in \mathbb{N}$ and let $\left(E,\|\cdot\|_{E}\right)$ be a real or complex Banach space. Following [4], we denote by $V\left(\mathbb{R}^{n}, E\right)$ the space of all functions $u \in C^{\infty}\left(\mathbb{R}^{n}, E\right)$ such that, for every non-empty bounded set $\Omega \subseteq \mathbb{R}^{n}$, one has

$$
\|u\|_{\Omega, E}:=\sup _{\alpha \in \mathbb{N}_{0}^{n}} \sup _{x \in \Omega}\left\|D^{\alpha} u(x)\right\|_{E}<\infty,
$$

where $D^{\alpha} u=\partial^{\alpha_{1}+\ldots+\alpha_{n}} u / \partial x_{1}^{\alpha_{1}} \ldots \partial x_{n}^{\alpha_{n}}, \alpha=\left(\alpha_{1}, \ldots, \alpha_{n}\right)$ and $\mathbb{N}_{0}=$ $\mathbb{N} \cup\{0\}$.

In the present paper, we are interested in the well-posedness in the space $V\left(\mathbb{R}^{n}, E\right)$ of the Cauchy problem

$$
\text { (1) } \begin{cases}D_{t}^{k} u+\sum_{j=0}^{k-1} \sum_{|\alpha| \leq m} A_{j, \alpha}\left(D_{t}^{j} D_{x}^{\alpha} u\right)=f & \text { in } \mathbb{R}^{n+1}, \\ D_{t}^{j} u(0, x)=\varphi_{j}(x) & \text { in } \mathbb{R}^{n}, j=0, \ldots, k-1,\end{cases}
$$

where each $A_{j, \alpha}$ is a given continuous linear operator from $E$ into itself. We denote by $\mathcal{L}(E)$ the space of all continuous linear operators from $E$ into

1991 Mathematics Subject Classification: Primary 35G10.

Key words and phrases: partial differential equations in Banach spaces, nilpotent operators. 
itself, endowed with the usual norm

$$
\|A\|_{\mathcal{L}(E)}=\sup _{\|v\|_{E} \leq 1}\|A(v)\|_{E} .
$$

Apparently, the only previous result on this subject is Theorem 1 of [4], where one assumes that

$$
\sum_{j=0}^{k-1} \sum_{|\alpha| \leq m}\left\|A_{j, \alpha}\right\|_{\mathcal{L}(E)}<1
$$

We wish here to prove another, independent result supposing that the operators $A_{j, \alpha}$ are nilpotent and pairwise commuting. However, a complete characterization of the well-posedness of the problem (1) in the space $V\left(\mathbb{R}^{n}, E\right)$ remains still unknown.

We believe that such a characterization should be quite difficult. To support this, we now discuss a particularly simple case which shows the peculiarity of working in the space $V\left(\mathbb{R}^{n}, E\right)$ rather than in the other spaces usually considered in the theory of linear partial differential equations.

Let $\sigma$ be a non-negative real number. Denote by $\Gamma^{(\sigma)}\left(\mathbb{R}^{2}\right)$ the (real) Gevrey class in $\mathbb{R}^{2}$ of index $\sigma$. That is to say, $\Gamma^{(\sigma)}\left(\mathbb{R}^{2}\right)$ is the class of all real functions $u \in C^{\infty}\left(\mathbb{R}^{2}\right)$ such that, for every non-empty bounded set $\Omega \subseteq \mathbb{R}^{2}$, one has

$$
\inf _{\lambda>0} \sup _{(\alpha, \beta) \in \mathbb{N}_{0}^{2}} \sup _{(t, x) \in \Omega}\left(\frac{1}{(\alpha+\beta) !}\right)^{\sigma} \lambda^{\alpha+\beta}\left|\frac{\partial^{\alpha+\beta} u(t, x)}{\partial t^{\alpha} \partial x^{\beta}}\right|<\infty .
$$

Recall, in particular, that $\Gamma^{(1)}\left(\mathbb{R}^{2}\right)$ coincides with the class of analytic functions in $\mathbb{R}^{2}$. Also, observe that $V\left(\mathbb{R}^{2}, \mathbb{R}\right) \subseteq \Gamma^{(0)}\left(\mathbb{R}^{2}\right)$.

Given a real number $a$, consider now the differential operator $P_{a}: C^{\infty}\left(\mathbb{R}^{2}\right)$ $\rightarrow C^{\infty}\left(\mathbb{R}^{2}\right)$ defined by

$$
P_{a}(u)=\frac{\partial^{k} u}{\partial t^{k}}+a \frac{\partial^{m} u}{\partial x^{m}}
$$

Then, according to the classical work of Malgrange [3], we have

$$
P_{a}\left(C^{\infty}\left(\mathbb{R}^{2}\right)\right)=C^{\infty}\left(\mathbb{R}^{2}\right) \quad \text { for every } a \in \mathbb{R} .
$$

Analogously, we have

$$
P_{a}\left(\Gamma^{(\sigma)}\left(\mathbb{R}^{2}\right)\right)=\Gamma^{(\sigma)}\left(\mathbb{R}^{2}\right) \quad \text { for every } a \in \mathbb{R} \text { and } \sigma \in[0,1[\cup([1, \infty[\cap \mathbb{Q}) .
$$

Precisely, this follows from Theorems 9.4 and 9.6 of [5] (see also p. 408 and pp. 467-468) when $\sigma \in[0,1[$, and from Theorem 4.1 of $[1]$ when $\sigma \in[1, \infty[\cap \mathbb{Q}$ (in the case $\sigma=1$ the result was previously proved in [2]).

Now, we come to the space $V\left(\mathbb{R}^{2}, \mathbb{R}\right)$ (for short $V\left(\mathbb{R}^{2}\right)$ ). On the basis of Theorem 4 of [4], we have

$$
P_{a}\left(V\left(\mathbb{R}^{2}\right)\right)=V\left(\mathbb{R}^{2}\right) \quad \text { if and only if } \quad a \neq \pm 1 .
$$


1. The result. Our result is the following.

Theorem 1. Let $k, n, m \in \mathbb{N}$, and let $\left\{A_{j, \alpha}\right\}_{j=0, \ldots, k-1, \alpha \in \mathbb{N}_{0}^{n},|\alpha| \leq m}$ be a family of pairwise commuting elements of $\mathcal{L}(E)$ such that for some $\bar{q} \in \mathbb{N}$ one has

$$
\left\|A_{j, \alpha}^{\bar{q}}\right\|_{\mathcal{L}(E)}=0 \quad \text { for each } j=0,1, \ldots, k-1 \text { and } \alpha \in \mathbb{N}_{0}^{n} \text { with }|\alpha| \leq m .
$$

Then for each $f \in V\left(\mathbb{R}^{n+1}, E\right)$ and each $\varphi_{0}, \varphi_{1}, \ldots, \varphi_{k-1} \in V\left(\mathbb{R}^{n}, E\right)$ there exists a unique function $u \in V\left(\mathbb{R}^{n+1}, E\right)$ such that for each $t \in \mathbb{R}$ and each $x \in \mathbb{R}^{n}$ one has

$$
\left\{\begin{array}{l}
D_{t}^{k} u(t, x)+\sum_{j=0}^{k-1} \sum_{|\alpha| \leq m} A_{j, \alpha}\left(D_{t}^{j} D_{x}^{\alpha} u(t, x)\right)=f(t, x), \\
D_{t}^{j} u(0, x)=\varphi_{j}(x), \quad j=0, \ldots, k-1 .
\end{array}\right.
$$

Moreover, if $p$ is the cardinality of the set $\left\{A_{j, \alpha}: A_{j, \alpha} \neq 0, j=0, \ldots, k-1\right.$, $\left.\alpha \in \mathbb{N}_{0}^{n},|\alpha| \leq m\right\}$ and $\bar{s}:=k^{2} p \bar{q}$, then for each bounded set $\Omega \subseteq \mathbb{R}^{n}$, and each $r \geq 0$ and $\lambda>0$, if one puts

$$
\sigma:=\max \left\{\lambda, \sum_{j=0}^{k-1} \sum_{|\alpha| \leq m} \lambda^{j-k+1}\left\|A_{j, \alpha}\right\|_{\mathcal{L}(E)}\right\},
$$

one has the following inequality:

$$
\begin{aligned}
& \max _{0 \leq i \leq k-1} \lambda^{-i}\left\|D_{t}^{i} u\right\|_{[-r, r] \times \Omega, E} \\
& \leq \min \left\{\left(\max _{0 \leq j \leq \bar{s}-1} \sigma^{j}\right) \max _{0 \leq i \leq k-1}\left(\lambda^{-i}\left\|\varphi_{i}\right\|_{\Omega, E}\right) e^{r \sigma}\right. \\
& +\left(\left(\max _{0 \leq j \leq \bar{s}-1} \sigma^{j}\right) r e^{r \sigma}+\sum_{j=0}^{\bar{s}-1} \sigma^{j}\right) \lambda^{1-k}\|f\|_{[-r, r] \times \Omega, E}, \\
& e^{r}\left(\left(\max _{0 \leq j \leq \bar{s}-1} \sigma^{j}\right)\left(\max _{0 \leq i \leq k-1} \lambda^{-i}\left\|\varphi_{i}\right\|_{\Omega, E}\right)\right. \\
& \left.\left.+\left(\sum_{j=0}^{\bar{s}-1} \sigma^{j}\right) \lambda^{1-k}\|f\|_{\{0\} \times \Omega, E}\right)\right\} .
\end{aligned}
$$

Before giving the proof of Theorem 1, we need some preliminary results.

Proposition 2. Let $A \in \mathcal{L}(E), B \in V(\mathbb{R}, E)$ and $v \in C^{1}(\mathbb{R}, E)$ be such that $\sum_{n=1}^{\infty}\left\|A^{n}\right\|_{\mathcal{L}(E)}<\infty$ and

$$
v^{\prime}(t)=A(v(t))+B(t) \quad \text { in } \mathbb{R} .
$$

Then $v \in V(\mathbb{R}, E)$ and for each $r \geq 0$ the following inequality holds: 
(3) $\|v\|_{[-r, r], E} \leq \min \left\{\left(\sup _{n \in \mathbb{N}_{0}}\left\|A^{n}\right\|_{\mathcal{L}(E)}\right)\|v(0)\|_{E} e^{r\|A\|_{\mathcal{L}(E)}}\right.$

$$
\begin{gathered}
+\left(\left(\sup _{n \in \mathbb{N}_{0}}\left\|A^{n}\right\|_{\mathcal{L}(E)}\right) r e^{r\|A\|_{\mathcal{L}(E)}}+\sum_{n=0}^{\infty}\left\|A^{n}\right\|_{\mathcal{L}(E)}\right)\|B\|_{[-r, r], E}, \\
\left.e^{r}\left(\left(\sup _{n \in \mathbb{N}_{0}}\left\|A^{n}\right\|_{\mathcal{L}(E)}\right)\|v(0)\|_{E}+\left(\sum_{n=0}^{\infty}\left\|A^{n}\right\|_{\mathcal{L}(E)}\right)\|B\|_{0, E}\right)\right\},
\end{gathered}
$$

where we put $\left\|A^{0}\right\|_{\mathcal{L}(E)}=1$.

P r o of. First, observe that $\sup _{n \in \mathbb{N}}\left\|A^{n}\right\|_{\mathcal{L}(E)} \leq \sum_{n=1}^{\infty}\left\|A^{n}\right\|_{\mathcal{L}(E)}<\infty$. Since $B \in C^{\infty}(\mathbb{R}, E)$ we get $v \in C^{\infty}(\mathbb{R}, E)$ and, arguing by induction, we have

$$
v^{(m)}(t)=A^{m}(v(t))+\sum_{j=1}^{m-1} A^{j}\left(B^{(m-j-1)}(t)\right)+B^{(m-1)}(t)
$$

for all $t \in \mathbb{R}$ and $m \in \mathbb{N}$ with $m \geq 2$. Now, fix any $r \geq 0$. We get $\left\|v^{(m)}(t)\right\|_{E}$

$$
\begin{aligned}
& \leq\left\|A^{m}\right\|_{\mathcal{L}(E)}\|v(t)\|_{E}+\sum_{j=1}^{m-1}\left\|A^{j}\right\|_{\mathcal{L}(E)}\left\|B^{(m-j-1)}(t)\right\|_{E}+\left\|B^{(m-1)}(t)\right\|_{E} \\
& \leq \sup _{n \in \mathbb{N}}\left\|A^{n}\right\|_{\mathcal{L}(E)} \sup _{t \in[-r, r]}\|v(t)\|_{E}+\left(\sum_{n=1}^{\infty}\left\|A^{n}\right\|_{\mathcal{L}(E)}+1\right)\|B\|_{[-r, r], E}
\end{aligned}
$$

for each $t \in[-r, r]$ and $m \geq 2$. It is easy to see that the last inequality also holds for $t \in[-r, r]$ and $m=1$. Hence

$\left\|v^{(m)}(t)\right\|_{E}$

$$
\leq \max \left\{1, \sup _{n \in \mathbb{N}}\left\|A^{n}\right\|_{\mathcal{L}(E)}\right\} \sup _{t \in[-r, r]}\|v(t)\|_{E}+\left(\sum_{n=0}^{\infty}\left\|A^{n}\right\|_{\mathcal{L}(E)}\right)\|B\|_{[-r, r], E}
$$

for each $t \in[-r, r]$ and $m \in \mathbb{N}_{0}$. Consequently, $v \in V(\mathbb{R}, E)$ and

(4) $\|v\|_{[-r, r], E}$

$$
\leq \sup _{n \in \mathbb{N}_{0}}\left\|A^{n}\right\|_{\mathcal{L}(E)} \sup _{t \in[-r, r]}\|v(t)\|_{E}+\left(\sum_{n=0}^{\infty}\left\|A^{n}\right\|_{\mathcal{L}(E)}\right)\|B\|_{[-r, r], E}
$$

for each $r \geq 0$. In particular, by (4) we get

$$
\|v\|_{0, E} \leq\left(\sup _{n \in \mathbb{N}_{0}}\left\|A^{n}\right\|_{\mathcal{L}(E)}\right)\|v(0)\|_{E}+\left(\sum_{n=0}^{\infty}\left\|A^{n}\right\|_{\mathcal{L}(E)}\right)\|B\|_{0, E} .
$$

By Proposition 2 of [4] we have $\|v\|_{[-r, r], E} \leq e^{r}\|v\|_{0, E}$, hence 
(5) $\quad\|v\|_{[-r, r], E}$

$$
\leq e^{r}\left(\left(\sup _{n \in \mathbb{N}_{0}}\left\|A^{n}\right\|_{\mathcal{L}(E)}\right)\|v(0)\|_{E}+\left(\sum_{n=0}^{\infty}\left\|A^{n}\right\|_{\mathcal{L}(E)}\right)\|B\|_{0, E}\right) .
$$

On the other hand, since $v(t)=v(0)+\int_{0}^{t} A(v(\tau)) d \tau+\int_{0}^{t} B(\tau) d \tau$ we get

$$
\|v(t)\|_{E} \leq\|v(0)\|_{E}+r\|B\|_{[-r, r], E}+\|A\|_{\mathcal{L}(E)}\left|\int_{0}^{t}\|v(\tau)\|_{E} d \tau\right|
$$

for every $t \in[-r, r]$. By Gronwall's lemma, we get

$$
\|v(t)\|_{E} \leq\left(\|v(0)\|_{E}+r\|B\|_{[-r, r], E}\right) e^{r\|A\|_{\mathcal{L}(E)}}
$$

for all $t \in[-r, r]$. By (4) and (6) we get

$$
\begin{aligned}
& \|v\|_{[-r, r], E} \\
& \left.\leq \sup _{n \in \mathbb{N}_{0}}\left\|A^{n}\right\|_{\mathcal{L}(E)}\right)\|v(0)\|_{E} e^{r\|A\|_{\mathcal{L}(E)}} \\
& \quad+\left(\left(\sup _{n \in \mathbb{N}_{0}}\left\|A^{n}\right\|_{\mathcal{L}(E)}\right) r e^{r\|A\|_{\mathcal{L}(E)}}+\sum_{n=0}^{\infty}\left\|A^{n}\right\|_{\mathcal{L}(E)}\right)\|B\|_{[-r, r], E} .
\end{aligned}
$$

Our claim follows easily from (5) and (7).

We point out that the operator $A$ satisfies $\sum_{n=1}^{\infty}\left\|A^{n}\right\|_{\mathcal{L}(E)}<\infty$ if, for instance, $\|A\|_{\mathcal{L}(E)}<1$ or if there is some $\bar{m} \in \mathbb{N}$ such that $A^{m}=0$ for all $m \geq \bar{m}$. When the former situation occurs, Proposition 2 reduces to Proposition 4 of [4], while in the latter case from Proposition 2 we get

(8)

$$
\begin{aligned}
& \|v\|_{[-r, r], E} \\
& \leq \min \left\{\left(\max _{0 \leq j \leq \bar{m}-1}\left\|A^{j}\right\|_{\mathcal{L}(E)}\right)\|v(0)\|_{E} e^{r\|A\|_{\mathcal{L}(E)}}\right. \\
& \quad+\left(\left(\max _{0 \leq j \leq \bar{m}-1}\left\|A^{j}\right\|_{\mathcal{L}(E)}\right) r e^{r\|A\|_{\mathcal{L}(E)}}+\sum_{j=0}^{\bar{m}-1}\left\|A^{j}\right\|_{\mathcal{L}(E)}\right)\|B\|_{[-r, r], E}, \\
& \left.\quad e^{r}\left(\left(\max _{0 \leq j \leq \bar{m}-1}\left\|A^{j}\right\|_{\mathcal{L}(E)}\right)\|v(0)\|_{E}+\left(\sum_{j=0}^{\bar{m}-1}\left\|A^{j}\right\|_{\mathcal{L}(E)}\right)\|B\|_{0, E}\right)\right\} .
\end{aligned}
$$

Proposition 3. Let $k \in \mathbb{N}$ with $k \geq 2$, and let $A_{0}, A_{1}, \ldots, A_{k-1} \in \mathcal{L}(E)$ be pairwise commuting operators. Assume that there exists $m^{*} \in \mathbb{N}$ such that $A_{j}^{m^{*}}=0$ for each $j=0,1, \ldots, k-1$. Let $\lambda>0$, and consider the operator $A: E^{k} \rightarrow E^{k}$ defined by

$$
A(y)=\left(\lambda y_{1}, \lambda y_{2}, \ldots, \lambda y_{k-1}, \sum_{j=0}^{k-1} \lambda^{j-k+1} A_{j}\left(y_{j}\right)\right)
$$

for each $y=\left(y_{0}, y_{1}, \ldots, y_{k-1}\right) \in E^{k}$. Then $A^{\bar{m}}=0$ for $\bar{m}=k^{2} m^{*}$. 
Pr o of. We divide the proof into several steps.

First step. Let $y=\left(y_{0}, y_{1}, \ldots, y_{k-1}\right) \in E^{k}$ and $s \in\{1, \ldots, k\}$ be fixed. Let us show that if one puts $A^{s}(y)=\left(x_{0}, x_{1}, \ldots, x_{k-1}\right)$, then the vector $x_{j}$ can be represented in the following way:

$$
x_{j}=\sum_{m=1}^{m_{j}} \mu_{j, m}(\lambda) A_{0}^{n_{j, m, 0}} A_{1}^{n_{j, m, 1}} \ldots A_{k-1}^{n_{j, m, k-1}}\left(y_{r(j, m)}\right)
$$

if $j=k-s, \ldots, k-1$ (with $m_{j} \geq 1$, and $\sum_{l=0}^{k-1} n_{j, m, l} \geq 1, r(j, m) \in$ $\{0,1, \ldots, k-1\}$ for each $\left.m=1, \ldots, m_{j}\right)$, and

$$
x_{j}=\lambda^{s} y_{j+s}
$$

if $j=0, \ldots, k-s-1$ (if $s<k$ ). To prove our claim, we argue by induction on $s$. Of course, our claim is true for $s=1$. Now, assume that it is true for $s=i$ (with $i<k$ ) and let us show that it remains true for $s=i+1$. By assumption, if we put $A^{i}(y)=\left(\widetilde{x}_{0}, \widetilde{x}_{1}, \ldots, \widetilde{x}_{k-1}\right)$, then we have

$$
\widetilde{x}_{j}=\sum_{m=1}^{\tilde{m}_{j}} \widetilde{\mu}_{j, m}(\lambda) A_{0}^{\tilde{n}_{j, m, 0}} A_{1}^{\tilde{n}_{j, m, 1}} \ldots A_{k-1}^{\tilde{n}_{j, m, k-1}}\left(y_{\tilde{r}(j, m)}\right)
$$

if $j=k-i, \ldots, k-1$ (with $\widetilde{m}_{j} \geq 1$, and $\sum_{l=0}^{k-1} \widetilde{n}_{j, m, l} \geq 1, \widetilde{r}(j, m) \in$ $\{0,1, \ldots, k-1\}$ for each $\left.m=1, \ldots, \widetilde{m}_{j}\right)$, and

$$
\widetilde{x}_{j}=\lambda^{i} y_{j+i}
$$

if $j=0, \ldots, k-i-1$. Put $A^{i+1}(y)=\left(w_{0}, w_{1}, \ldots, w_{k-1}\right)$. We get

$$
\begin{aligned}
w_{k-1}= & \sum_{d=0}^{k-1} \lambda^{d-k+1} A_{d}\left(\widetilde{x}_{d}\right) \\
= & \sum_{d=0}^{k-i-1} \lambda^{d-k+1} A_{d}\left(\lambda^{i} y_{d+i}\right) \\
& +\sum_{d=k-i}^{k-1} \lambda^{d-k+1} A_{d}\left(\sum_{m=1}^{\tilde{m}_{d}} \widetilde{\mu}_{d, m}(\lambda) A_{0}^{\tilde{n}_{d, m, 0}} A_{1}^{\tilde{n}_{d, m, 1}} \ldots A_{k-1}^{\tilde{n}_{d, m, k-1}}\left(y_{\tilde{r}(d, m)}\right)\right) \\
= & \sum_{d=0}^{k-i-1} \lambda^{d-k+i+1} A_{d}\left(y_{d+i}\right) \\
& +\sum_{d=k-i}^{k-1} \sum_{m=1}^{\tilde{m}_{d}} \lambda^{d-k+1} \widetilde{\mu}_{d, m}(\lambda) A_{d}\left(A_{0}^{\tilde{n}_{d, m, 0}} A_{1}^{\tilde{n}_{d, m, 1}} \ldots A_{k-1}^{\tilde{n}_{d, m, k-1}}\left(y_{\tilde{r}(d, m)}\right)\right),
\end{aligned}
$$

hence it is easily seen that $w_{k-1}$ is of the form (9). Now, let $j \in\{k-i-1$, 
$\ldots, k-2\}$. We get

$w_{j}=\lambda \widetilde{x}_{j+1}=\lambda \sum_{m=1}^{\tilde{m}_{j+1}} \widetilde{\mu}_{j+1, m}(\lambda) A_{0}^{\tilde{n}_{j+1, m, 0}} A_{1}^{\tilde{n}_{j+1, m, 1}} \ldots A_{k-1}^{\tilde{n}_{j+1, m, k-1}}\left(y_{\tilde{r}(j+1, m)}\right)$,

hence $w_{j}$ is of the form (9) even for $j=k-i-1, \ldots, k-2$. Thus, if $i=k-1$, our claim follows. If $i<k-1$, for each $j=0, \ldots, k-i-2$ we have

$$
w_{j}=\lambda \widetilde{x}_{j+1}=\lambda^{i+1} y_{j+i+1} \text {, }
$$

as desired.

Second step. We prove that for each fixed $y=\left(y_{0}, y_{1}, \ldots, y_{k-1}\right) \in$ $E^{k}$ and $s \in \mathbb{N}$, if we put $A^{s k}(y)=\left(z_{0}, z_{1}, \ldots, z_{k-1}\right)$, then for each $j \in$ $\{0,1, \ldots, k-1\}$ the vector $z_{j}$ can be represented in the following way:

$$
z_{j}=\sum_{m=1}^{b_{j}} \sigma_{j, m}(\lambda) A_{0}^{p_{j, m, 0}} A_{1}^{p_{j, m, 1}} \ldots A_{k-1}^{p_{j, m, k-1}}\left(y_{v(j, m)}\right)
$$

with $b_{j} \geq 1$ and $\sum_{l=0}^{k-1} p_{j, m, l} \geq s, v(j, m) \in\{0,1, \ldots, k-1\}$ for each $m=$ $1, \ldots, b_{j}$. Again, we argue by induction. First, we observe that by the first part of the proof, if we put $A^{k}(y)=\left(u_{0}, u_{1}, \ldots, u_{k-1}\right)$, then for each $j=$ $0,1, \ldots, k-1$ the vector $u_{j}$ can be represented in the form

$$
u_{j}=\sum_{m=1}^{\bar{m}_{j}} \bar{\mu}_{j, m}(\lambda) A_{0}^{\bar{n}_{j, m, 0}} A_{1}^{\bar{n}_{j, m, 1}} \ldots A_{k-1}^{\bar{n}_{j, m, k-1}}\left(y_{\bar{r}(j, m)}\right)
$$

with $\bar{m}_{j} \geq 1$ and $\sum_{l=0}^{k-1} \bar{n}_{j, m, l} \geq 1, \bar{r}(j, m) \in\{0,1, \ldots, k-1\}$ for each $m=1, \ldots, \bar{m}_{j}$, hence our claim is true for $s=1$. Assume that it is true for $s=i$, and let us show that it is true for $s=i+1$. Thus, if we put $A^{i k}(y)=\left(\widehat{z}_{0}, \widehat{z}_{1}, \ldots, \widehat{z}_{k-1}\right)$, then for each $j=0,1, \ldots, k-1$ the vector $\widehat{z}_{j}$ can be represented in the following way:

$$
\widehat{z}_{j}=\sum_{m=1}^{\hat{b}_{j}} \widehat{\sigma}_{j, m}(\lambda) A_{0}^{\hat{p}_{j, m, 0}} A_{1}^{\hat{p}_{j, m, 1}} \ldots A_{k-1}^{\hat{p}_{j, m, k-1}}\left(y_{\hat{v}(j, m)}\right)
$$

with $\widehat{b}_{j} \geq 1$ and $\sum_{l=0}^{k-1} \widehat{p}_{j, m, l} \geq i, \widehat{v}(j, m) \in\{0,1, \ldots, k-1\}$ for each $m=1, \ldots, \widehat{b}_{j}$. From the first part of the proof, if we put $A^{(i+1) k}(y)=$ $A^{k}\left(A^{i k}(y)\right)=\left(\widehat{w}_{0}, \widehat{w}_{1}, \ldots, \widehat{w}_{k-1}\right)$, then for each $j=0,1, \ldots, k-1$ we have

$$
\widehat{w}_{j}=\sum_{m=1}^{\hat{m}_{j}} \widehat{\mu}_{j, m}(\lambda) A_{0}^{\hat{n}_{j, m, 0}} A_{1}^{\hat{n}_{j, m, 1}} \ldots A_{k-1}^{\hat{n}_{j, m, k-1}}\left(\widehat{z}_{\hat{r}(j, m)}\right)
$$

with $\widehat{m}_{j} \geq 1$ and $\sum_{l=0}^{k-1} \widehat{n}_{j, m, l} \geq 1, \widehat{r}(j, m) \in\{0,1, \ldots, k-1\}$ for each 
$m=1, \ldots, \widehat{m}_{j}$. By $(10)$, for each $j=0,1, \ldots, k-1$ we get

$$
\begin{aligned}
\widehat{w}_{j} & =\sum_{m=1}^{\hat{m}_{j}} \sum_{d=1}^{\hat{b}_{\hat{r}(j, m)}} \widehat{\mu}_{j, m}(\lambda) \widehat{\sigma}_{\hat{r}(j, m), d}(\lambda) \\
& \cdot A_{0}^{\hat{n}_{j, m, 0}+\hat{p}_{\hat{r}(j, m), d, 0}} A_{1}^{\hat{n}_{j, m, 1}+\hat{p}_{\hat{r}(j, m), d, 1}} \ldots A_{k-1}^{\hat{n}_{j, m, k-1}+\hat{p}_{\hat{r}(j, m), d, k-1}}\left(y_{\hat{v}(\hat{r}(j, m), d)}\right) .
\end{aligned}
$$

Since for each $m \in\left\{1, \ldots, \widehat{m}_{j}\right\}$ and $d \in\left\{1, \ldots, b_{\hat{r}(j, m)}\right\}$ we have $\sum_{l=0}^{k-1} \widehat{n}_{j, m, l}$ $+\widehat{p}_{\hat{r}(j, m), d, l} \geq i+1$, our claim follows.

Third step. We claim that $A^{k^{2} m^{*}}=0$. To see this, choose any $y=$ $\left(y_{0}, y_{1}, \ldots, y_{k-1}\right) \in E^{k}$. If we put $A^{k^{2} m^{*}}(y)=\left(\widetilde{w}_{0}, \widetilde{w}_{1}, \ldots, \widetilde{w}_{k-1}\right)$, from the second part of the proof we see that for each $j=0,1, \ldots, k-1$ the vector $\widetilde{w}_{j}$ can be represented in the following way:

$$
\widetilde{w}_{j}=\sum_{m=1}^{\tilde{b}_{j}} \widetilde{\sigma}_{j, m}(\lambda) A_{0}^{\tilde{p}_{j, m, 0}} A_{1}^{\tilde{p}_{j, m, 1}} \ldots A_{k-1}^{\tilde{p}_{j, m, k-1}}\left(y_{\tilde{v}(j, m)}\right)
$$

with $\widetilde{b}_{j} \geq 1$ and $\sum_{l=0}^{k-1} \widetilde{p}_{j, m, l} \geq k m^{*}, \widetilde{v}(j, m) \in\{0,1, \ldots, k-1\}$ for each $m=1, \ldots, \widetilde{b}_{j}$. Now, it is easy to see that for each fixed $j \in\{0,1, \ldots, k-1\}$ and $m \in\left\{1, \ldots, \widetilde{b}_{j}\right\}$ there exists $\widetilde{l} \in\{0,1, \ldots, k-1\}$ such that $\widetilde{p}_{j, m, \tilde{l}} \geq m^{*}$. Hence, we conclude that $\widetilde{w}_{j}=0$ for all $j=0,1, \ldots, k-1$. This completes the proof.

Proposition 4. Let $k \in \mathbb{N}$ and let $A_{0}, A_{1}, \ldots, A_{k-1} \in \mathcal{L}(E)$ be pairwise commuting operators. Assume that there exists $m^{*} \in \mathbb{N}$ such that $A_{j}^{m^{*}}=$ 0 for each $j=0,1, \ldots, k-1$. Then for each $B \in V(\mathbb{R}, E)$ and for each $w_{0}, w_{1}, \ldots, w_{k-1} \in E$, there exists a unique $v \in V(\mathbb{R}, E)$ such that

$$
\begin{cases}v^{(k)}(t)=\sum_{j=0}^{k-1} A_{j}\left(v^{(j)}(t)\right)+B(t) & \text { for all } t \in \mathbb{R}, \\ v^{(j)}(0)=w_{j} & \text { for } j=0,1, \ldots, k-1 .\end{cases}
$$

Moreover, if $\bar{m}:=k^{2} m^{*}$, then for each fixed $r \geq 0$ and $\lambda>0$, if one puts

$$
c_{A}=\max \left\{\lambda, \sum_{j=0}^{k-1} \lambda^{j-k+1}\left\|A_{j}\right\|_{\mathcal{L}(E)}\right\}
$$

one has

$$
\begin{aligned}
& \max _{0 \leq i \leq k-1} \lambda^{-i}\left\|v^{(i)}\right\|_{[-r, r], E} \\
& \leq \min \left\{\left(\max _{0 \leq j \leq \bar{m}-1} c_{A}^{j}\right) \max _{0 \leq i \leq k-1} \lambda^{-i}\left\|w_{i}\right\|_{E} e^{r c_{A}}\right.
\end{aligned}
$$




$$
\begin{aligned}
& +\left(\left(\max _{0 \leq j \leq \bar{m}-1} c_{A}^{j}\right) r e^{r c_{A}}+\sum_{j=0}^{\bar{m}-1} c_{A}^{j}\right) \lambda^{1-k}\|B\|_{[-r, r], E}, \\
& \left.e^{r}\left(\left(\max _{0 \leq j \leq \bar{m}-1} c_{A}^{j}\right) \max _{0 \leq i \leq k-1} \lambda^{-i}\left\|w_{i}\right\|_{E}+\left(\sum_{j=0}^{\bar{m}-1} c_{A}^{j}\right) \lambda^{1-k}\|B\|_{0, E}\right)\right\} .
\end{aligned}
$$

Proof. If $k=1$, our claim follows by Picard-Lindelöf's theorem and Proposition 2. Now, let $k \geq 2$, and consider the space $E^{k}$ endowed with the norm

$$
\|y\|_{E^{k}}=\max _{0 \leq j \leq k-1}\left\|y_{i}\right\|_{E},
$$

where $y=\left(y_{0}, y_{1}, \ldots, y_{k-1}\right)$. Fix $\lambda>0$, and let $A: E^{k} \rightarrow E^{k}$ be defined by setting

$$
A\left(y_{0}, y_{1}, \ldots, y_{k-1}\right)=\left(\lambda y_{1}, \lambda y_{2}, \ldots, \lambda y_{k-1}, \sum_{j=0}^{k-1} \lambda^{j-k+1} A_{j}\left(y_{j}\right)\right)
$$

for each $y \in E^{k}$. Now, observe that for each $y \in E^{k}$ one has

$$
\|A(y)\|_{E^{k}} \leq \max \left\{\lambda, \sum_{j=0}^{k-1} \lambda^{j-k+1}\left\|A_{j}\right\|_{\mathcal{L}(E)}\right\}\|y\|_{E^{k}}=c_{A}\|y\|_{E^{k}}
$$

Thus, $A \in \mathcal{L}\left(E^{k}\right)$ and $\|A\|_{\mathcal{L}\left(E^{k}\right)} \leq c_{A}$. By Proposition 3 one has $A^{\bar{m}}=0$, where $\bar{m}=k^{2} m^{*}$. By Picard-Lindelöf's theorem, there exists a unique $v \in$ $C^{k}(\mathbb{R}, E)$ such that

$$
\begin{cases}v^{(k)}(t)=\sum_{j=0}^{k-1} A_{j}\left(v^{(j)}(t)\right)+B(t) & \text { for } t \in \mathbb{R} \\ v^{(j)}(0)=w_{j} & \text { for all } j=0,1, \ldots, k-1 .\end{cases}
$$

Let $\Gamma: \mathbb{R} \rightarrow E^{k}$ and $\omega: \mathbb{R} \rightarrow E^{k}$ be defined by setting for each $t \in \mathbb{R}$,

$$
\begin{aligned}
\Gamma(t) & =\left(0,0, \ldots, \lambda^{1-k} B(t)\right), \\
\omega(t) & =\left(v(t), \lambda^{-1} v^{\prime}(t), \lambda^{-2} v^{\prime \prime}(t), \ldots, \lambda^{1-k} v^{(k-1)}(t)\right) .
\end{aligned}
$$

It is easy to see that $\Gamma \in V\left(\mathbb{R}, E^{k}\right), \omega \in C^{1}\left(\mathbb{R}, E^{k}\right)$ and

$$
\omega^{\prime}(t)=A(\omega(t))+\Gamma(t) \quad \text { for all } t \in \mathbb{R} \text {. }
$$

By Proposition 2 we get $\omega \in V\left(\mathbb{R}, E^{k}\right)$, hence $v \in V(\mathbb{R}, E)$. Moreover, (8) gives

$$
\begin{aligned}
\max _{0 \leq i \leq k-1} \lambda^{-i}\left\|v^{(i)}\right\|_{[-r, r], E} & =\|\omega\|_{[-r, r], E^{k}} \\
& \leq \min \left\{\left(\max _{0 \leq j \leq \bar{m}-1}\left\|A^{j}\right\|_{\mathcal{L}\left(E^{k}\right)}\right)\|\omega(0)\|_{E^{k}} e^{r\|A\|_{\mathcal{L}\left(E^{k}\right)}}\right.
\end{aligned}
$$




$$
\begin{aligned}
& +\left(\left(\max _{0 \leq j \leq \bar{m}-1}\left\|A^{j}\right\|_{\mathcal{L}\left(E^{k}\right)}\right) r e^{r\|A\|_{\mathcal{L}\left(E^{k}\right)}}+\sum_{j=0}^{\bar{m}-1}\left\|A^{j}\right\|_{\mathcal{L}\left(E^{k}\right)}\right)\|\Gamma\|_{[-r, r], E^{k}}, \\
& \left.e^{r}\left(\left(\max _{0 \leq j \leq \bar{m}-1}\left\|A^{j}\right\|_{\mathcal{L}\left(E^{k}\right)}\right)\|\omega(0)\|_{E^{k}}+\left(\sum_{j=0}^{\bar{m}-1}\left\|A^{j}\right\|_{\mathcal{L}\left(E^{k}\right)}\right)\|\Gamma\|_{0, E^{k}}\right)\right\}
\end{aligned}
$$

for every $r \geq 0$. Since $\left\|A^{j}\right\|_{\mathcal{L}\left(E^{k}\right)} \leq\|A\|_{\mathcal{L}\left(E^{k}\right)}^{j} \leq c_{A}^{j}$ for each $j=1, \ldots, \bar{m}-1$, our claim follows at once.

Proof of Theorem 1. First, we denote by $F_{1}, \ldots, F_{p}$ the elements of the set $\left\{A_{j, \alpha}: A_{j, \alpha} \neq 0, j=0, \ldots, k-1, \alpha \in \mathbb{N}_{0}^{n},|\alpha| \leq m\right\}$. Fix $f \in$ $V\left(\mathbb{R}^{n+1}, E\right)$ and $\varphi_{0}, \varphi_{1}, \ldots, \varphi_{k-1} \in V\left(\mathbb{R}^{n}, E\right)$, where the space $V\left(\mathbb{R}^{n}, E\right)$ will be considered with any norm $\|\cdot\|_{\Omega, E}$. For each $j=0,1, \ldots, k-1$, $v \in V\left(\mathbb{R}^{n}, E\right)$ and $x \in \mathbb{R}^{n}$, let

$$
T_{j}(v)(x)=-\sum_{|\alpha| \leq m} A_{j, \alpha}\left(D^{\alpha} v(x)\right) .
$$

By Proposition 6 of [4] we have $T_{j} \in \mathcal{L}\left(V\left(\mathbb{R}^{n}, E\right)\right)$ and $\left\|T_{j}\right\|_{\mathcal{L}\left(V\left(\mathbb{R}^{n}, E\right)\right)} \leq$ $\sum_{|\alpha| \leq m}\left\|A_{j, \alpha}\right\|_{\mathcal{L}(E)}$. Consider the problem

$$
\begin{cases}\omega^{(k)}(t)=\sum_{j=0}^{k-1} T_{j}\left(\omega^{(j)}(t)\right)+\Psi^{-1}(f)(t) & \text { in } \mathbb{R} \\ \omega^{(j)}(0)=\varphi_{j} & \text { for } j=0,1, \ldots, k-1\end{cases}
$$

where $\Psi: V\left(\mathbb{R}, V\left(\mathbb{R}^{n}, E\right)\right) \rightarrow V\left(\mathbb{R}^{n+1}, E\right)$ is the function defined as in Proposition 3 of [4]. Namely, $\Psi(g)(t, x)=g(t)(x)$ for $g \in V\left(\mathbb{R}, V\left(\mathbb{R}^{n}, E\right)\right)$, $t \in \mathbb{R}$, and $x \in \mathbb{R}^{n}$. Now, it is easily seen that the operators $T_{j}$ are pairwise commuting. We claim that

$$
\left\|T_{j}^{p \bar{q}}\right\|_{\mathcal{L}\left(V\left(\mathbb{R}^{n}, E\right)\right)}=0 \quad \text { for each } j=0,1, \ldots, k-1 .
$$

To see this, let $j \in\{0,1, \ldots, k-1\}, v \in V\left(\mathbb{R}^{n}, E\right)$ and $x \in \mathbb{R}^{n}$ be fixed, and let $\left\{A_{j, \alpha(j, i)}\right\}_{i=1}^{s_{j}}$ be the elements of the family $\left\{A_{j, \alpha}\right\}_{\alpha \in \mathbb{N}_{0}^{n},|\alpha| \leq m}$ that are different from the origin of $\mathcal{L}(E)$. Thus, we have

$$
T_{j}(v)(x)=-\sum_{i=1}^{s_{j}} A_{j, \alpha(j, i)}\left(D^{\alpha(j, i)} v(x)\right) .
$$

Now we show that for each $h \in \mathbb{N}$ the vector $T_{j}^{h}(v)(x)$ can be represented as follows:

$$
\begin{aligned}
T_{j}^{h}(v)(x) & =(-1)^{h} \sum_{l=1}^{b(h)} A_{j, \alpha(j, 1)}^{r(h, l, 1)} A_{j, \alpha(j, 2)}^{r(h, l, 2)} \ldots \\
\ldots & A_{j, \alpha\left(j, s_{j}\right)}^{r\left(h, l, s_{j}\right)}\left(D^{r(h, l, 1) \alpha(j, 1)+r(h, l, 2) \alpha(j, 2)+\ldots+r\left(h, l, s_{j}\right) \alpha\left(j, s_{j}\right)} v(x)\right)
\end{aligned}
$$


for suitable $b(h) \in \mathbb{N}$ and $r(h, l, 1), r(h, l, 2), \ldots, r\left(h, l, s_{j}\right) \in \mathbb{N}$ with

$$
\sum_{i=1}^{s_{j}} r(h, l, i)=h \quad \text { for each } l \in\{1, \ldots, b(h)\} .
$$

We argue by induction. Of course, our claim is true for $h=1$, with $r(1, l, d)=$ 1 if $d=l$, while $r(1, l, d)=0$ if $d \neq l$. Now, assume that our claim is true for some $h \in \mathbb{N}$. We have

$$
\begin{aligned}
& T_{j}^{h+1}(v)(x) \\
& =T_{j}\left(T_{j}^{h}(v)\right)(x)=-\sum_{i=1}^{s_{j}} A_{j, \alpha(j, i)}\left(D^{\alpha(j, i)} T_{j}^{h}(v)(x)\right) \\
& =\sum_{i=1}^{s_{j}}(-1)^{h+1} \sum_{l=1}^{b(h)} A_{j, \alpha(j, 1)}^{r(h, l, 1)} A_{j, \alpha(j, 2)}^{r(h, l, 2)} \ldots \\
& \quad \ldots A_{j, \alpha\left(j, s_{j}\right)}^{r\left(h, l, s_{j}\right)} A_{j, i}\left(D^{\alpha(j, i)} D^{r(h, l, 1) \alpha(j, 1)+r(h, l, 2) \alpha(j, 2)+\ldots+r\left(h, l, s_{j}\right) \alpha\left(j, s_{j}\right)} v(x)\right) .
\end{aligned}
$$

Now, it is easy to see that $T_{j}^{h+1}(v)(x)$ is also of the form (14). Hence, our claim is true for $h+1$, hence it is true for all $h \in \mathbb{N}$. In particular, if $h=p \bar{q}$, the representation (14) holds, with $\sum_{i=1}^{s_{j}} r(h, l, i)=p \bar{q}$ for each fixed $l \in\{1, \ldots, b(p \bar{q})\}$. Observe that, for each fixed $l \in\{1, \ldots, b(p \bar{q})\}$, we have

$$
A_{j, \alpha(j, 1)}^{r(p \bar{q}, l, 1)} A_{j, \alpha(j, 2)}^{r(p \bar{q}, l, 2)} \ldots A_{j, \alpha\left(j, s_{j}\right)}^{r\left(p \bar{q}, l, s_{j}\right)}=F_{1}^{q_{1}} \ldots F_{p}^{q_{p}}
$$

with

$$
\sum_{i=1}^{p} q_{i}=\sum_{d=1}^{s_{j}} r(p \bar{q}, l, d)=p \bar{q} .
$$

Of course, this implies that there exists $i \in\{1, \ldots, p\}$ such that $q_{i} \geq \bar{q}$, hence $F_{i}^{q_{i}}=0$. Therefore, for each fixed $l \in\{1, \ldots, b(\bar{q} h)\}$, the operator

$$
A_{j, \alpha(j, 1)}^{r(p \bar{q}, l, 1)} A_{j, \alpha(j, 2)}^{r(p \bar{q}, l, 2)} \ldots A_{j, \alpha\left(j, s_{j}\right)}^{r\left(p \bar{q}, l, s_{j}\right)}
$$

identically vanishes, hence $T_{j}^{h}(v)(x)=0_{E}$. The arbitrariness of $v \in V\left(\mathbb{R}^{n}, E\right)$ and $x \in \mathbb{R}^{n}$ gives

$$
\left\|T_{j}^{p \bar{q}}\right\|_{\mathcal{L}\left(V\left(\mathbb{R}^{n}, E\right)\right)}=0 \quad \text { for each } j=0,1, \ldots, k-1 .
$$

By Proposition 4 there exists a unique $\omega \in V\left(\mathbb{R}, V\left(\mathbb{R}^{n}, E\right)\right)$ satisfying (13). Now, if $t \in \mathbb{R}$ and $x \in \mathbb{R}^{n}$, we have 


$$
\begin{aligned}
\omega^{(k)}(t)(x) & =\Psi\left(\omega^{(k)}\right)(t, x)=\sum_{j=0}^{k-1} \Psi\left(T_{j} \circ \omega^{(j)}\right)(t, x)+f(t, x) \\
& =-\sum_{j=0}^{k-1} \sum_{|\alpha| \leq m} A_{j, \alpha}\left(D^{\alpha} \omega^{(j)}(t)(x)\right)+f(t, x), \\
\omega^{(j)}(0)(x) & =\varphi_{j}(x) \quad \text { for all } j=0,1, \ldots, k-1 .
\end{aligned}
$$

If we put $u=\Psi(\omega)$, observing that by Proposition 3 of [4] we have $D_{t}^{j} \Psi(\omega)=$ $\Psi\left(D^{j} \omega\right)$, for each $t \in \mathbb{R}$ and $x \in \mathbb{R}^{n}$ we get

$$
\left\{\begin{array}{l}
D_{t}^{k} u(t, x)+\sum_{j=0}^{k-1} \sum_{|\alpha| \leq m} A_{j, \alpha}\left(D_{t}^{j} D_{x}^{\alpha} u(t, x)\right)=f(t, x), \\
D_{t}^{j} u(0, x)=\varphi_{j}(x) \text { for all } j=0,1, \ldots, k-1 .
\end{array}\right.
$$

Hence, $u$ is a solution of problem (2). Conversely, reasoning as in [4] one can show that if $\widetilde{u}$ solves (2), then $\widetilde{u}=u$. By Proposition 4, if $\bar{s}:=k^{2} p \bar{q}, r \geq 0$ and $\lambda>0$, we get

$$
\begin{aligned}
& \max _{0 \leq i \leq k-1} \lambda^{-i}\left\|\omega^{(i)}\right\|_{[-r, r], V\left(\mathbb{R}^{n}, E\right)} \\
& \leq \min \left\{\left(\max _{0 \leq j \leq \bar{s}-1} \sigma^{j}\right) \max _{0 \leq i \leq k-1}\left(\lambda^{-i}\left\|\varphi_{i}\right\|_{V\left(\mathbb{R}^{n}, E\right)}\right) e^{r \sigma}\right. \\
&+\left(\left(\max _{0 \leq j \leq \bar{s}-1} \sigma^{j}\right) r e^{r \sigma}+\sum_{j=0}^{\bar{s}-1} \sigma^{j}\right) \lambda^{1-k}\left\|\Psi^{-1}(f)\right\|_{[-r, r], V\left(\mathbb{R}^{n}, E\right)}, \\
& e^{r}\left(\left(\max _{0 \leq j \leq \bar{s}-1} \sigma^{j}\right) \max _{0 \leq i \leq k-1}\left(\lambda^{-i}\left\|\varphi_{i}\right\|_{V\left(\mathbb{R}^{n}, E\right)}\right)\right. \\
&\left.\left.+\left(\sum_{j=0}^{\bar{s}-1} \sigma^{j}\right) \lambda^{1-k}\left\|\Psi^{-1}(f)\right\|_{0, V\left(\mathbb{R}^{n}, E\right)}\right)\right\} \\
& \leq \min \left\{\left(\max _{0 \leq j \leq \bar{s}-1} \sigma^{j}\right) \max _{0 \leq i \leq k-1}\left(\lambda^{-i}\left\|\varphi_{i}\right\|_{\Omega, E}\right) e^{r \sigma}\right. \\
&+\left(\left(\max _{0 \leq j \leq \bar{s}-1} \sigma^{j}\right) r e^{r \sigma}+\sum_{j=0} \sigma^{j}\right) \lambda^{1-k}\|f\|_{[-r, r] \times \Omega, E}, \\
& e^{r}\left(\left(\max _{0 \leq j \leq \bar{s}-1} \sigma^{j}\right) \max _{0 \leq i \leq k-1}\left(\lambda^{-i}\left\|\varphi_{i}\right\|_{\Omega, E}\right)\right. \\
&\left.\left.+\left(\sum_{j=0}^{\bar{s}-1} \sigma^{j}\right) \lambda^{1-k}\|f\|_{\{0\} \times \Omega, E}\right)\right\},
\end{aligned}
$$

where $\Omega$ is any non-empty bounded subset of $\mathbb{R}^{n}$. Since

$$
\left\|D_{t}^{i} u\right\|_{[-r, r] \times \Omega, E}=\left\|\omega^{(i)}\right\|_{[-r, r], V\left(\mathbb{R}^{n}, E\right)},
$$

our claim follows. 
To conclude, we now present a simple example of application of Theorem 1 to integro-differential equations. Let $Y \subseteq \mathbb{R}^{m}(m \in \mathbb{N})$ be a nonempty compact set. Following [4], denote by $V_{0}\left(\mathbb{R}^{n} \times Y\right)$ the space of all functions $u: \mathbb{R}^{n} \times Y \rightarrow \mathbb{R}$ such that $u(\cdot, y) \in C^{\infty}\left(\mathbb{R}^{n}\right)$ for each $y \in Y$, $D_{x}^{\alpha} u \in C^{0}\left(\mathbb{R}^{n} \times Y\right)$ for each $\alpha \in \mathbb{N}_{0}^{n}$ and

$$
\sup _{\alpha \in \mathbb{N}_{0}^{n}} \sup _{(x, y) \in \Omega \times Y}\left|D_{x}^{\alpha} u(x, y)\right|<\infty
$$

for each bounded set $\Omega \subseteq \mathbb{R}^{n}$. Also recall ([4], Proposition 8) that if, for $u \in V\left(\mathbb{R}^{n}, C^{0}(Y)\right)\left(C^{0}(Y)\right.$ is endowed with the usual sup-norm), $\Psi_{n}(u)$ denotes the function, from $\mathbb{R}^{n} \times Y$ into $\mathbb{R}$, defined by

$$
\Psi_{n}(u)(x, y)=u(x)(y) \quad\left(x \in \mathbb{R}^{n}, y \in Y\right),
$$

then $\Psi_{n}(u) \in V_{0}\left(\mathbb{R}^{n} \times Y\right)$, the mapping $u \rightarrow \Psi_{n}(u)$ is surjective, and, for each $\alpha \in \mathbb{N}_{0}^{n}$, one has $D_{x}^{\alpha} \Psi_{n}(u)=\Psi_{n}\left(D^{\alpha} u\right)$.

TheOREM 2. Let $k, n, m \in \mathbb{N}$ and let $\left\{g_{j, \alpha}\right\}_{j=0, \ldots, k-1, \alpha \in \mathbb{N}_{0}^{n},|\alpha| \leq m}$ be a family of continuous real functions on $Y$ each of which satisfies $\int_{Y} g_{j, \alpha}(y) d y$ $=0$. Then, for each $f \in V_{0}\left(\mathbb{R}^{n+1} \times Y\right)$ and $\varphi_{0}, \varphi_{1}, \ldots, \varphi_{k-1} \in V_{0}\left(\mathbb{R}^{n} \times Y\right)$, there exists a unique function $u \in V_{0}\left(\mathbb{R}^{n+1} \times Y\right)$ such that, for each $t \in \mathbb{R}$, $x \in \mathbb{R}^{n}$ and $y \in Y$, one has

$$
\left\{\begin{array}{l}
D_{t}^{k} u(t, x, y)+\sum_{j=0}^{k-1} \sum_{|\alpha| \leq m}\left(\int_{Y} D_{t}^{j} D_{x}^{\alpha} u(t, x, \xi) d \xi\right) g_{j, \alpha}(y)=f(t, x, y), \\
D_{t}^{j} u(0, x, y)=\varphi_{j}(x, y), \quad j=0, \ldots, k-1 .
\end{array}\right.
$$

Proof. For each $j=0, \ldots, k-1, \alpha \in \mathbb{N}_{0}^{n}$, let $A_{j, \alpha}$ be the element of $\mathcal{L}\left(C^{0}(Y)\right)$ defined by putting

$$
A_{j, \alpha}(v)(y)=\left(\int_{Y} v(\xi) d \xi\right) g_{j, \alpha}(y)
$$

for all $v \in C^{0}(Y)$ and $y \in Y$. Clearly, $A_{j, \alpha} \circ A_{j^{\prime}, \alpha^{\prime}}=A_{j^{\prime}, \alpha^{\prime}} \circ A_{j, \alpha}=0$. Consequently, by Theorem 1 , there exists a unique function $w \in V\left(\mathbb{R}^{n+1}, C^{0}(Y)\right)$ such that, for each $t \in \mathbb{R}$ and $x \in \mathbb{R}^{n}$, one has

$$
\left\{\begin{array}{l}
D_{t}^{k} w(t, x)+\sum_{j=0}^{k-1} \sum_{|\alpha| \leq m} A_{j, \alpha}\left(D_{t}^{j} D_{x}^{\alpha} w(t, x)\right)=\Psi_{n+1}^{-1}(f)(t, x), \\
D_{t}^{j} w(0, x)=\Psi_{n}^{-1}\left(\varphi_{j}\right)(x), \quad j=0, \ldots, k-1 .
\end{array}\right.
$$

Then the function $u=\Psi_{n+1}(w)$ satisfies the conclusion.

\section{References}

[1] L. Cattabriga, On the surjectivity of differential polynomials on Gevrey spaces, Rend. Sem. Mat. Univ. Politec. Torino (1983), special issue on "Linear partial and pseudodifferential operators", 81-89. 
[2] L. Cattabriga and E. De Giorgi, Una dimostrazione diretta dell'esistenza di soluzioni analitiche nel piano reale di equazioni a derivate parziali a coefficienti costanti, Boll. Un. Mat. Ital. (4) 4 (1971), 1015-1027.

[3] B. Malgrange, Existence et approximation des solutions des équations aux dérivées partielles et des équations de convolution, Ann. Inst. Fourier (Grenoble) 6 (1955-56), 271-355.

[4] B. Ricceri, On the well-posedness of the Cauchy problem for a class of linear partial differential equations of infinite order in Banach spaces, J. Fac. Sci. Univ. Tokyo Sect. IA Math. 38 (1991), 623-640.

[5] F. Trèves, Linear Partial Differential Equations with Constant Coefficients, Gordon and Breach, 1966.

Department of Mathematics

University of Messina

98166 Sant'Agata-Messina, Italy

E-mail: cubiotti@mat520.unime.it

Reçu par la Rédaction le 30.9.1995 